\title{
PARTICLES AND FIELDS \\ IN THE MAGNETOSPHERE
}

by

Brian J. O'Brien

\section{Department of Space' ience Rice Universily \\ Houston, Texas}

Invited review paper/presented to the National Academy of Sciences Annual Meeting, April 26, 1967, Washington, D.C.
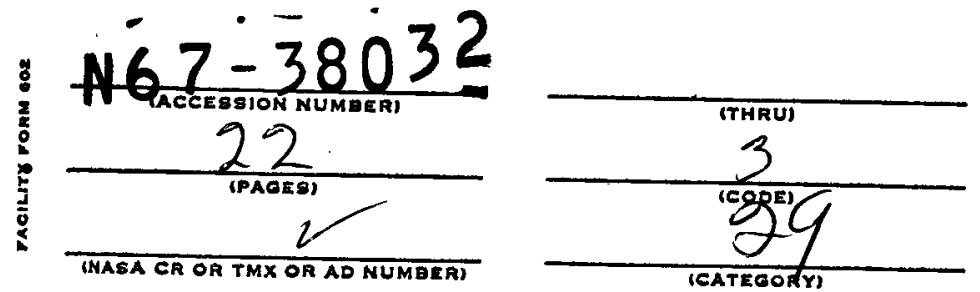
PARTICLES AND FIELDS IN THE MAGNETOSPHERE

\author{
by \\ Brian J. O'Brien \\ Department of Space science \\ Rice University \\ Houston, Texas
}

\title{
INTRODUCTION
}

The most extensively-studied space discipline of the postSputnik period is that of the "particles and fields" in the upper environment of the earth. Some 700 instruments on over 100 spacecraft have been used to make measurements of these phenomena. Consequently, over one billion relevant measurements have now been made in space, and an appreciable proportion of these were made during the period of the quiet sun, i.e. during the IQSY.

However, because of long lead times and uncertain launch dates in space flights, it is difficult to isolate IQSY activities per se. This review therefore is intended to summarize the present knowledge of magnetospheric particles and fields.

The need for an international coordinated program (such as the IQSY) has of course been recognized on several occasions. This need can be shown to be particularly true in studies of auroral and magnetospheric phenomena, since these phenomena are neither controllable nor reproducible, as are many laboratory measurements. Thus a concerted world-wide study of individual phenomena - such as was permitted and encouraged by the IQSY can yield far more worthwhile scientific results than the equivalent number of uncoordinated studies of separate "events". 
HISTORICAL GENERAL REVIEW

In the 1930's, Chapman and Ferraro [1932] after noting that great "world-wide" auroras occurred a day or two after a very large solar flare, speculated that the energy that sustained the auroras was supplied by corpuscular radiation emitted in the solar outburst. They considered only the rare "great" auroras, and envisaged the geomagnetic field as hollowing out a cavity in this fast-streaming flow of solar particles.

Subsequently, Parker [1959] suggested that this solarcorpuscular flow might be continuous and due simply to the immersion of the earth in the supersonically-expanding hot atmosphere of the sun. This plasma flow came to be called the "solar wind". It was also recognized that auroras occur continuously [O'Brien, 1967] and so the relationship of auroras and the solar--wind as its ultimate source of energy seemed even closer, although no direct theoretical causal links were established.

Figure 1 illustrates crudely the comparison between the magnetospheric concepts of the 1950's and one of those of the 1960's. Theoretical ideas and direct space measurements have developed a concept shown in more detail in Figure 2. The supersonic solar wind, travelling at around Mach 5, is obstructed by the geomagnetic field, so that an enormous collisionless shock front is set up (see Figure 2). Behind the shock front the solar wind particles are thermalized. Due to the fight for control between plasmas and local magnetic fields, the thermalized solar wind sweeps back the geomagnetic field into a cometlike tail behind the earth (see Figure 2). The length of this tail has been estimated theoretically, and estimates differ by factors of a thousand or more, but it does appear that the tail reaches beyond the orbit of the moon. 


\section{Auroras}

Auroras are amongst the most spectacular of geophysical phenomena, with their tremendous variations in shape, brightness, color and time and height. During the IGY, rocket flights into visual auroras demonstrated that most of their energy came from the kinetic energy of electrons of some 1 to $10 \mathrm{kev}$ as they bombarded and were absorbed in the atmosphere at altitudes of some 100 to $150 \mathrm{~km}$. Subsequently, through the IQSY, direct probing of the magnetosphere with rockets and satellites has sought to find the source of these auroral electrons and the protons that sometimes accompany them.

Unfortunately, however, the ultimate acceleration mechanisms that generate these auroral particles remain still largely unknown. However, it does appear, as sketched in Figure 2, that auroras form a pivotal role in the battle for supremacy between the solar-wind particles whose kinetic-energy density far exceeds that of the interplanetary magnetic field, and the innermost regions of the magnetosphere, where the strong geomagnetic fields "traps" Van Allen charged particles.

It is interesting to put into context the average amount of power dissipated world-wide in auroral phenomena. The average power is about 1000 Megawatts, or about $10 \%$ of the worldwide electric power dissipated by mankind. However, the total power brought by the solar wind to the magnetospheric surface is so large that only about $1 \%$ of it need be tapped to sustain auroras. No theory has yet been developed to indicate whether this $1 \%$ efficiency factor is "reasonable" or not. 
The Magnetosphere as a Plasma Laboratory

Besides the basic research potentialities in space-borne studies of auroras and the magnetosphere, it is very important for the pragmatist to regard it as a vast plasma laboratory. As such, it offers to the physicist the opportunity to study plasma phenomena on a scale impossible to simulate in the laboratory.

Now, it is well-known that in laboratory attempts to harness fusion processès to produce a practical source of electrical power, the practical limit is to the duration of stability of the "fusion" process. In general, the stability fails when wave-particle interactions take place in manners not thoroughly understood.

In auroras, as well, violent auroral instabilities are associated with intense wave-particle interactions that are even less understood. For example, many measurements demonstrate the occurrence of auroral "hiss", i.e. electromagnetic radiation in the VLF (audio) range, when visual auroras are bright. There are numerous other examples, both in the laboratory and in the magnetosphere, where waves and particles jointly interact, with sometimes one manifestation (e.g. waves) extracting energy from the particles, e.g. as in the case of the cyclotron resonance, and with the reverse taking place on other occasions, e.g. as in Landau damping. A few such examples are as shown in Figure 3.

Now, it is not intended to pursue this analogy too far because the scaling factors between the laboratory plasmas and the magnetospheric plasmas are so great. For example, typical plasma densities are some $10^{12}$ particles $\mathrm{cm}^{-3}$ and some 10 particles $\mathrm{cm}^{-3}$ respectively. Consequently in the laboratory one is generally dealing with a collision-dominated process, whereas most magnetospheric phenomena are considered as "collisionless". 
Nevertheless, it is important to note the fundamental pure plasma research opportunities opened up by exploration of the magnetosphere, and it is to be hoped that more plasma physicists will address themselves to such problems.

\section{Van Allen (trapped) Radiation}

Probably the single most spectacular space discovery of the post-Sputnik period remains that of the discovery of geomagnetically-trapped energy charged particles, subsequently called Van Allen radiation [Van Allen, 1961]. Figure 4 is an artist's concept showing, if you like, the evolution - to use the word loosely - from the IGY to the IQSY. You may remember seeing at various times drawings of the Van Allen radiation belts, an inner zone and outer zone. The reason they were drawn as an inner zone and outer zone is because the early experiments were fairly primitive Geiger tubes - necessarily so then - and they respond only to higher energy particles. These were mainly protons in the "inner" zone and electrons in the instable "outer" zone.

There thus developed this concept of two different zones, and various theoretical speculations followed as to them having different originsand losses. In fact in the following years, as more sophisticated particle detectors have been flown in the magnetosphere, we have found that the whole domain is populated by particles of varying energies and varying intensities. It is no longer at all clear what the commonality of source and loss mechanisms may be, except that it is known that at low altitudes near the equatorial plane attrition by coulomb collisions with atmospheric constituents is the dominant loss mechanism. This 'finding resulted from study of the gradual depletion of the artificial radiation belt caused by $\beta$-decay of fission debris from the July 9, 1962 high-altitude detonation of the 1.4 Megaton "Starfish" nuclear device (e.g. see O'Brien, 1967). 
It is equally clear however, that at higher altitudes and latitudes, the loss mechanisms are much stronger. They may, in part, be attributed to wave-particle interactions (e.g. see Kennel and Petschek, 1966) which appear to set an upper bound on the fluxes of stably-trapped particles. Another such upper bound has been mentioned briefly earlier, in that the particle kinetic energy density should be only a fraction (say 0.1) of the magnetic energy density for stable trapping to be sustained.

Two theories dominate the concepts of the cause of van Allen radiation. One is the so-called cosmic-ray neutron albedo, wherein it is known that energetic cosmic rays that hit the earth's atmosphere produce upwards-moving neutrons that may suffer $\beta$-decay in the magnetosphere, and may then inject electrons and protons at such angles that they will be trapped. This durable theory is now in general disrepute due to the weakness of the source, but it is a possible explanation of the very energetic ( $100 \mathrm{Mev}$ ) protons of the inner zone.

The other theory to which considerable attention is now being paid is the adiabatic diffusion theory, wherein it is assumed that charged particles "cross" magnetic lines of force, entering into regions of stronger geomagnetic fields so that betatron acceleration of the particles can occur (see Nakada and Mead, 1965). While the phenomenon can qualitatively explain several observed phenomenon in the Van Allen radiation, numerous problems remain in quantitative solutions as well as in details of the processes whereby charged particles are "injected" into the magnetosphere so that subsequent diffusion can accelerate them.

Our knowledge of the innermost regions of the Van Allen zone has reached the point where computer programs have been developed to calculate particle dosage of a satellite or an astronaut flying in a particular orbit at a particular time 
[Vette; et al., 1966]. At higher altitudes, the great variability of the particle fluxes with time and space prohibit any such orderly predictions.

\section{The Plasmapause}

Very-low frequency (VLF) electromagnetic radiation called a "whistler" is produced by a lightning stroke and dispersion of the different frequencies as the radiation travels through the magnetosphere ducted essentially along the magnetic field lines. Since the dispersion depends on the properties of the medium, e.g. the electron density, one can use these whistlers to probe the upper magnetosphere.

One of the most striking results has been the detection of the "plasmapause", and abrupt 10 to 100 fold decrease in electron density (Figure 5) at an altitude above $\sim 3$ earth radii [Carpenter, 1966]. More recently, Frank \{1967\} has reported that most of the residual electrons (density $(\sim 10 / \mathrm{Cc})$ on occasions may have an average energy of several hundred electron volts.

This introduces once again the fascinating problems of the interrelation of waves and charged particles. The propagation and properties of magnetospheric waves depend on the properties of the medium. In particular, for example, whether the wave loses energy to the particles or whether it energizes them depends on the energy of the particles as well as their density and hence the velocity and also the frequency of the waves. studies have been made in a preliminary way of some of these interactions (e.g. Figure 6) and it seems clear that a considerable advance in understanding of magnetospheric phenomena will result from coordinated in situ measurements of particles and fields, where the latter include not only magnetic fields but the much-neglected electric fields. 
Furthermore, it would appear that the plasmapause may indicate the boundary between plasma corotating with the earth and that which is involved in convective flow around the earth (e.g. see Nishida, 1966; Axford and Hines, 1961). The relevance of this hypothesis to the unknown sources of auroral radiation is not clear.

\section{Magnetic Fields}

Just as the spatial and temporal characteristics of magnetospheric energetic particles have been mapped in great detail, so have these characteristics of the geomagnetic field itself (e.g. see Cahill and Amazeen, 1963; Ness, 1965; and Fairchild and Ness, 1967). As a consequence, new model computations have been made for the "real" geomagnetic field, i.e. that which takes into account not only the magnetic field generated within the solid earth, but also externally-generated fields. Of these "external" fields most study has been concentrated on the ring current and on the tail of the geomagnetic field (e.g. see Ness review, 1967).

Indeed, the geomagnetic field morphology studies, whose accuracy and reliability were delayed for so long awaiting "magnetically-clean" spacecraft, are now comparable in sophistication and details to the energetic-particle studies. One can sketch (e.g. as in Figure 2) the postulated magnetospheric configuration.

The next major advances to be made are in studies of electric fields and of electric- and magnetic-waves in the magnetosphere. While there are preliminary measurements of these phenomena, (e.g. see Ness review, 1967) the complexity of making accurate and reliable measurements is extreme, due in large part to problems associated with plasmas sheaths around a detector or spacecraft, coupling of the antenna to the medium, and so on. 
It appears likely that significant scientific discoveries will result from appropriate technological advances - provided that experimentalists and theorists alike not stray down incorrect paths due to inadequate experimental protection against such potential sources of error as mentioned above.

\section{SUMMARY DISCUSSIONS}

It is convenient to summarize our knowledge of particle fluxes in the near-earth environment by the sketches of Figures 7 and 8 showing the wide variability in fluxes of particles of the solar wind, thermalized solar wind, auroras and Van Allen radiation. One of the principal single problems, of course, is how the relatively low-energy of solar-wind particles may be "concentrated" on fewer very high energy auroral or Van Allen particles, if indeed this process even occurs.

Figure 2 has illustrated some of the remarkable changes in our concept of the upper magnetosphere, an understanding achieved in many ways through coordinated programs such as the IGY and the IQSY. There seems little doubt - after viewing the two versions in Figure 1 - that even the configuration of

Figure 2 will be drawn differently after further space exploration. (Indeed there are some who would claim it nẹeds redrawing immediately).

The most noteworthy discoveries, as mentioned above, all follow from the fact that the earth's magnetosphere is like unto a vast plasma laboratory, wherein collisionless and other processes are occurring on a scale impossible to simulate in the laboratory, but from whose study we may hope to learn some of the fundamentals of plasma processes.

In spite of a bilition space measurements, we remain ignorant of such phenomena as the cause(s) of auroral and Van Allen radiation, and their ultimate source, e.g. were these particles 
once on the sun? From more definitive measurements and theories, and in particular from a deeper understanding of the fundamental plasma processes operative near the earth, it may be presumed that such understanding will come, and that furthermore it may have direct relevance to harnessing fusion processes in the laboratory for practical purposes. 


\section{REFERENCES}

Axford; W. I., and C. O. Hines, "A Unifying Theory of HighLatitude Geophysical Phenomena and Geomagnetic Storms", Can. J. Phys., 39, 1433-1464 (1961).

Cahil1, I., and P. G. Amazeen, "The Boundary of the Geomagnetic Field," J. Geophys. Res., 68, 1835-1844 (1963).

Carpenter, D. L., "Whistler Evidence of a 'Knee' in the Magnetosphere - 1; Temporal Variations in the Position of the Knee and Some Evidence on Plasma Motions Near the Knee,"J. Geophys. Res., 71, 693-709 (1966).

Chapman, S., and V. C. A. Ferraro, "A New Theory of Magnetic Storms", Terr. Mag. Atmos. Elec., 36, 77-97, 171-186 (1931); $147-156,421-429(1932)$.

Fairfield, D. H., and N. F. Ness, "Magnetic Field Measurements with the IMP-2 Satellite", J. Geophys. Res., 72, 2379-2402 (1967) .

Frank, L. A., "Initial Observations of Low-Energy Electrons in the Earth's Magnetosphere with OGO-3", J. Geophys. Res., 72, 185-195 (1967).

Kennel, C. F., and H. E. Petschek, "Limit on Stably Trapped Particle Fluxes", J. Geophys. Res.' 7i, 1-28, (1966).

Nakada, M. P. and G. D. Mead, "Diffusion of Protons in the outer Radiation Belt", J. Geophys. Res., 70, 4777-4791 (1965).

Ness, N. F., "The Earth's Magnetic Tail", J. Geophys. Res., 70, 2989-3005 (1965).

Ness, N. F., "Observations of the Solar wind Interaction with the Geomagnetic Field: Conditions Quiet", Solar-Terrestrial Physics, ed. Newman/King, Academic Press, Inc. (Iondon) (1967). 
Nishida, A., "Formation of Plasmapause, or Magnetospheric Plasma Knee by the Combined Action of Magnetospheric Convection and Plasma Escape from the Tail", J. Geophys. Res., 71, 56695679 (1966).

O'Brien, B. J., "Interrelations of Energetic Charged particles in the Magnetosphere", Solar-Terrestrial Physics, ed. Newman/ King, Academic Press, Inc. (London), Chapter VI, (1967).

Parker, E. N., "Extension of the Solar Corona into Interplanetary Space," J. Geophys. Res. 64, 1675-1681 (1959).

Parks, George K., "Spatial Characteristics of Auroral-Zone X-Ray Microbursts," J. Geophys. Res., 72, 215-226 (1967).

Van Allen, J. A., First public lecture on the discovery of geomagnetically trapped radiation, transcript of remarks as delivered on May 1, 1958 to the National Academy of Sciences, washington, D. C., IGY Satellite Report, No. 13, January 1961, IGY World Data Center A, Rockets and Satellites, National Academy of Sciences, National Research Council.

Vette, James I.. Antonio B. Lucero and Jon A. Wright, Models of the Trapped Radiation Environment, Vol. 2, "Inner and outer Zone Electrons", NASA SP-3024 (1966).

White, R. Stephen, "The Earth's Radiation Belts," Physics Today, 19, 25-38 (October 1966). 


\section{FIGURE CAPTIONS}

Figure 1: Comparison of sketches of the magnetosphere as essentially what might have been depicted just prior to the IGY (on the left) and during the IQSY (on the right). [From O'Brien, 1967].

Figure 2: Detailed sketch of the magnetosphere as envisaged from some magnetospheric models which depict the magnetic fields as "slowly" merging. Other sketches can be made with equal validity at this time but with significant differences in detail. [From O'Brien, 1967].

Figure. 3: A few samples of potential interactions between waves and particles in the magnetosphere or in the plasma laboratory.

Figure 4: Artist's concept of the magnetosphere. Note in particular the "inner" and "outer" Van Allen belts, now not treated as separate entities. [From White, 1966].

Figure 5: Illustration of the plasmapause, showing the abrupt decrease in density of thermal electrons at some $4 R_{E}$. [From Carpenter, 1966].

Figure 6: Illustration of simultaneous occurrence of magnetic pulsations and microbursts of auroral X-Rays. [From Parks, 1967].

Figure 7: Sketch to illustrate ranges of electron fluxes and spectra that may be encountered in the magnetosphere and environment.

Figure 8: Same as Figure 7 but for protons. 


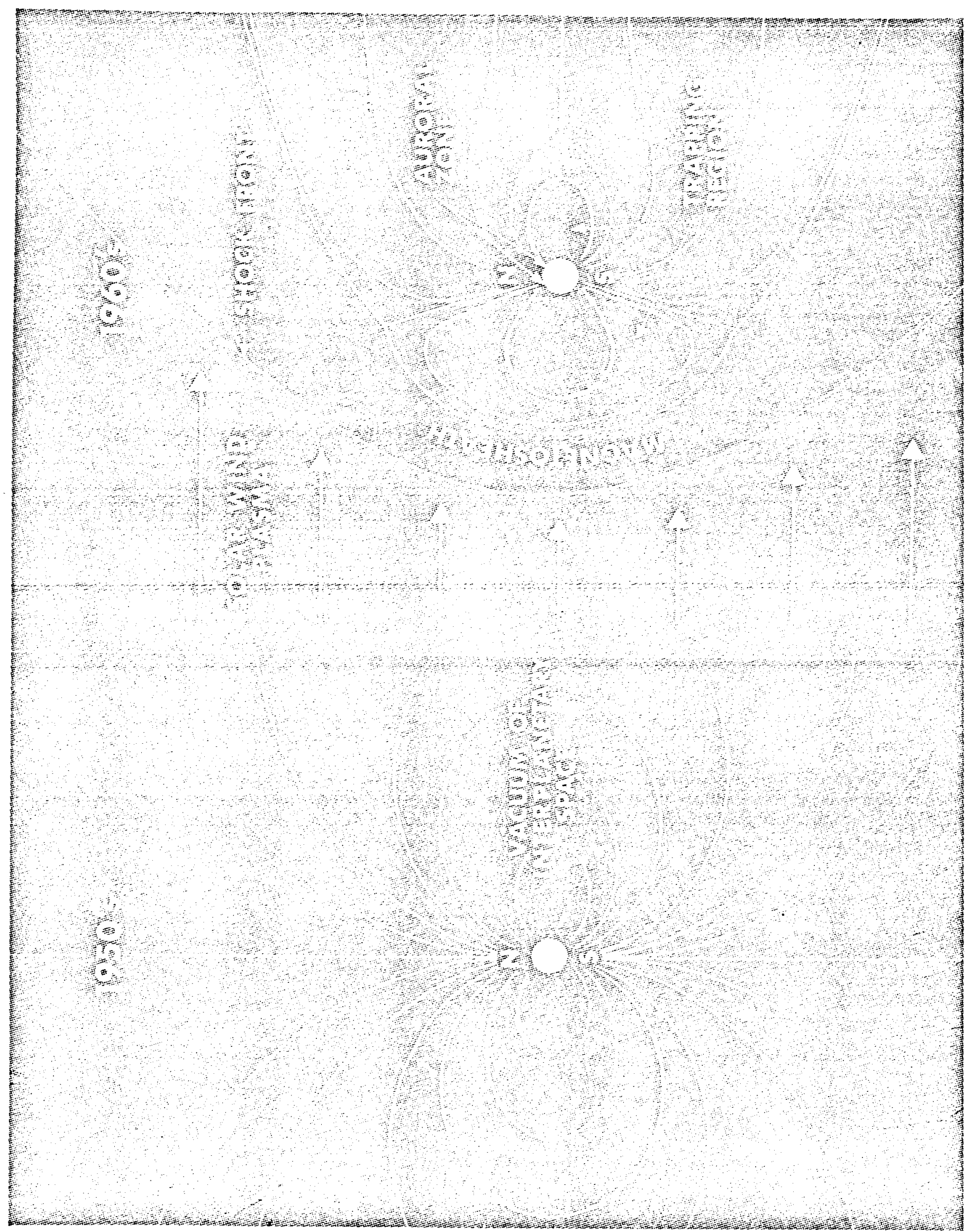




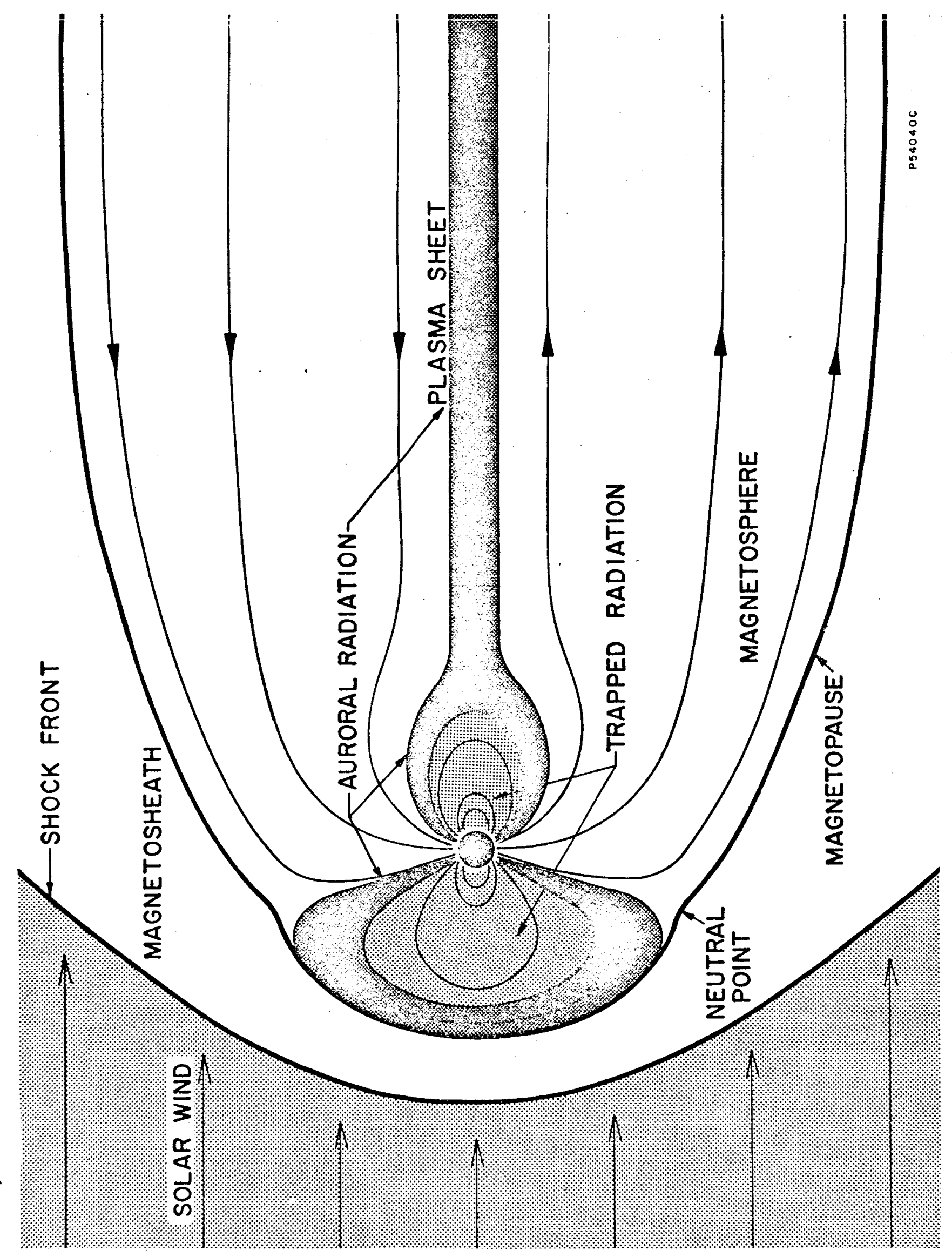



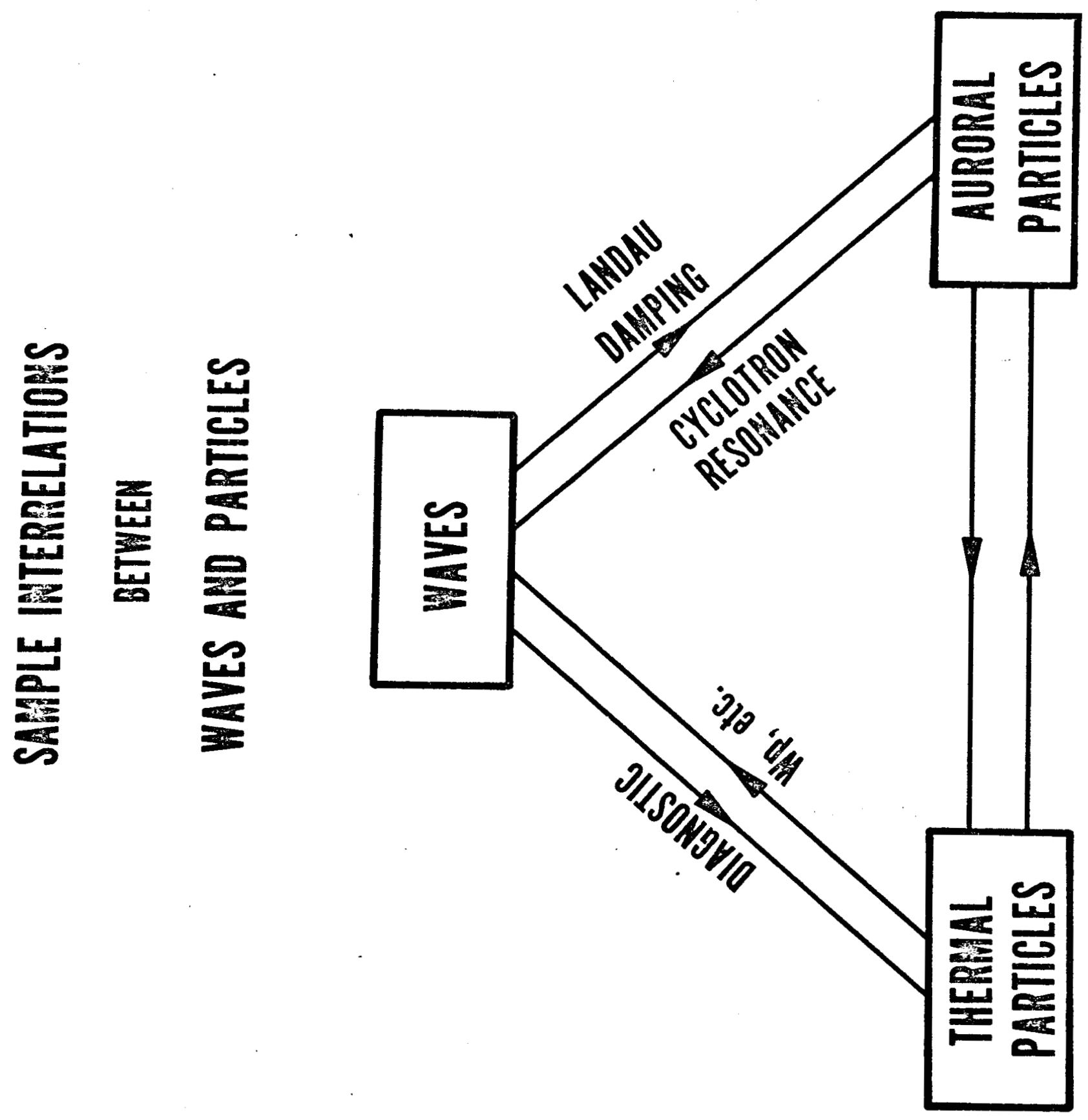


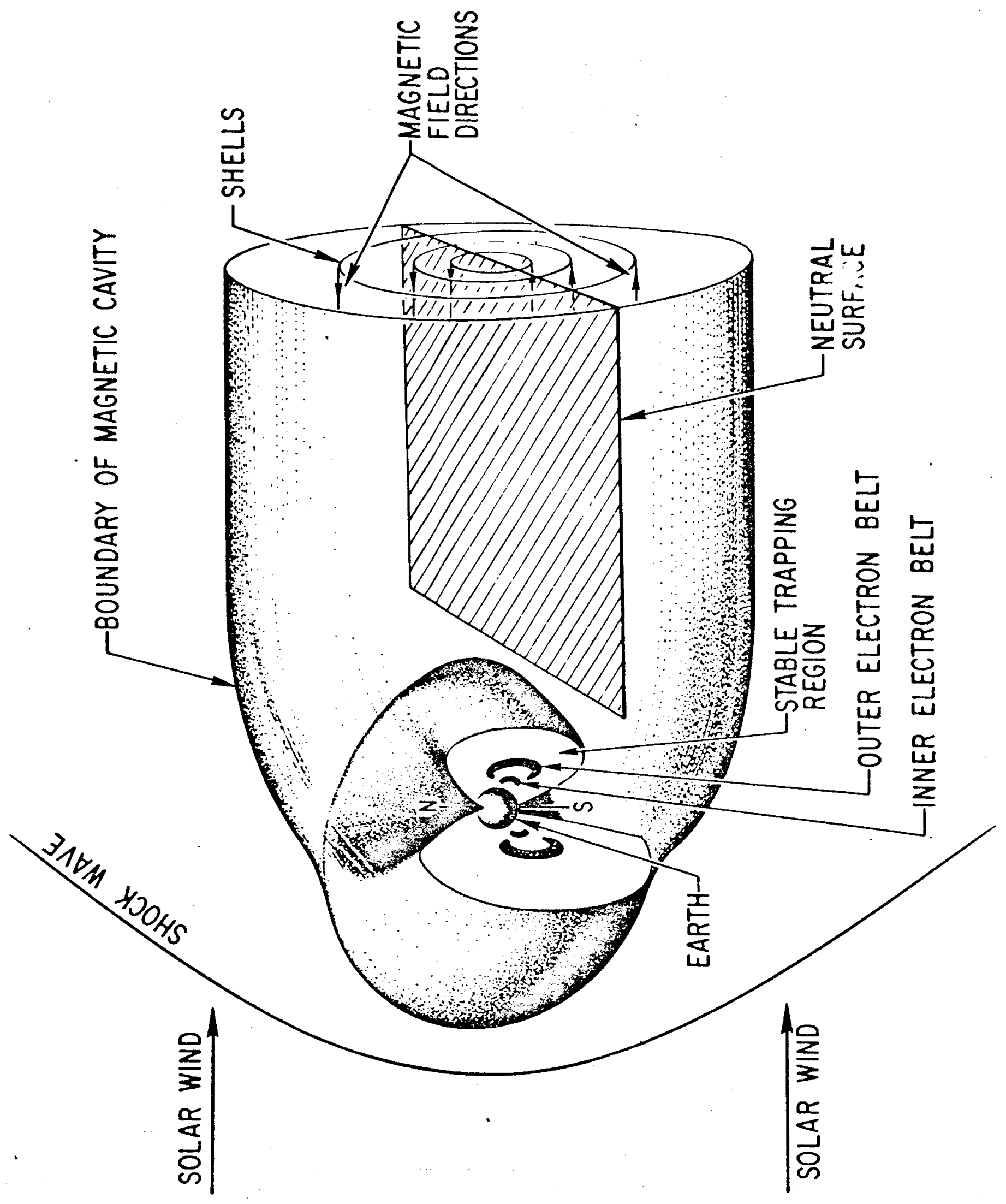




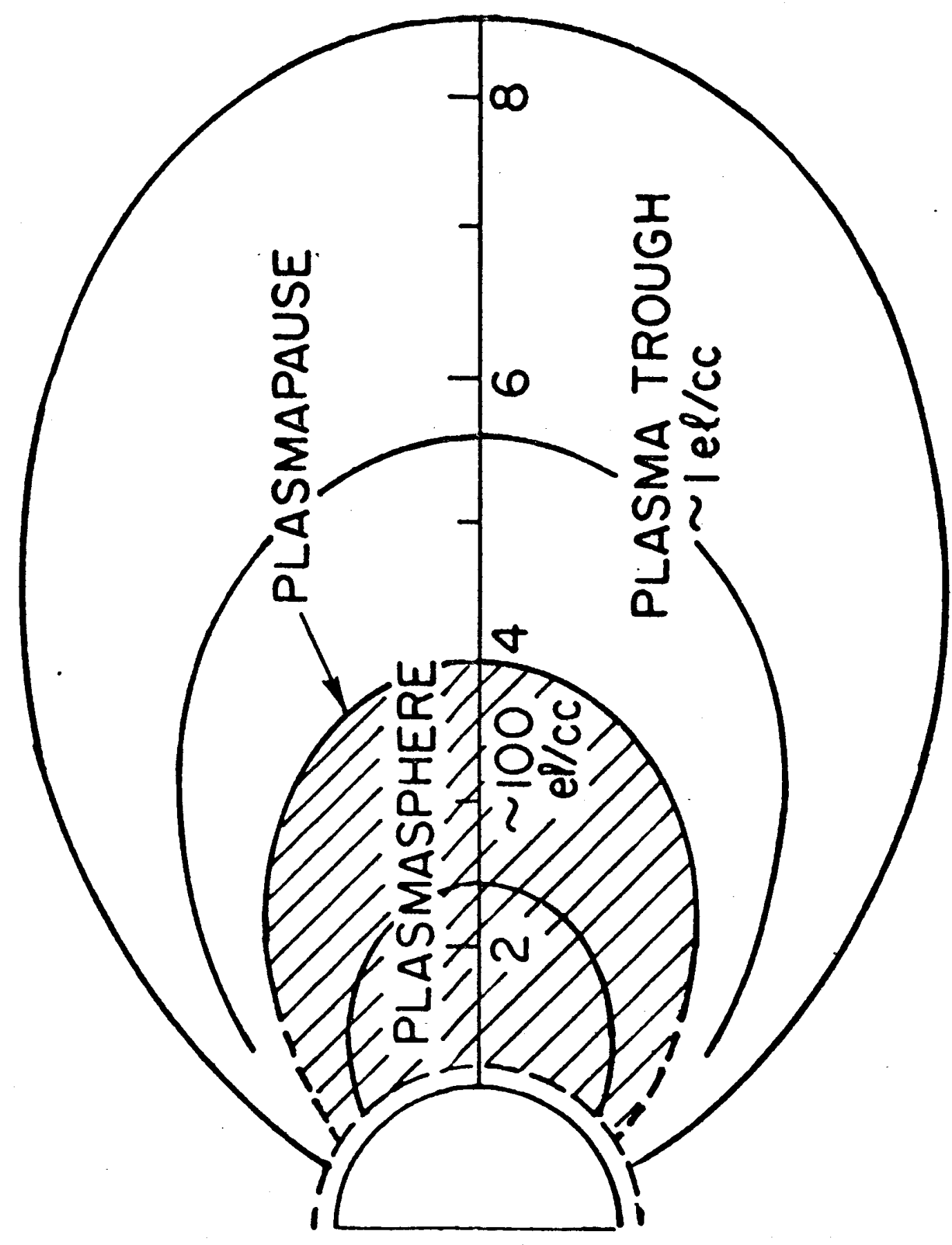




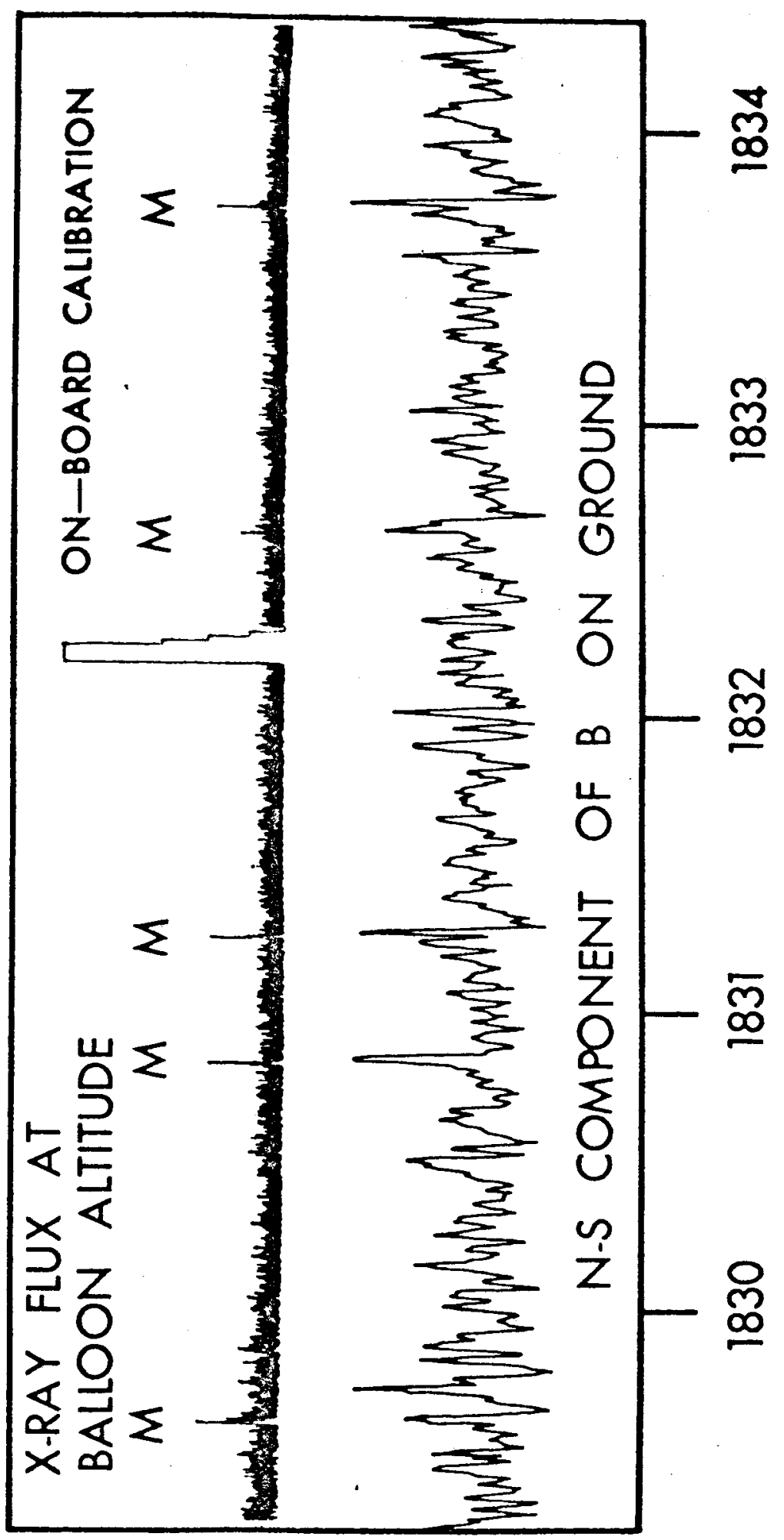




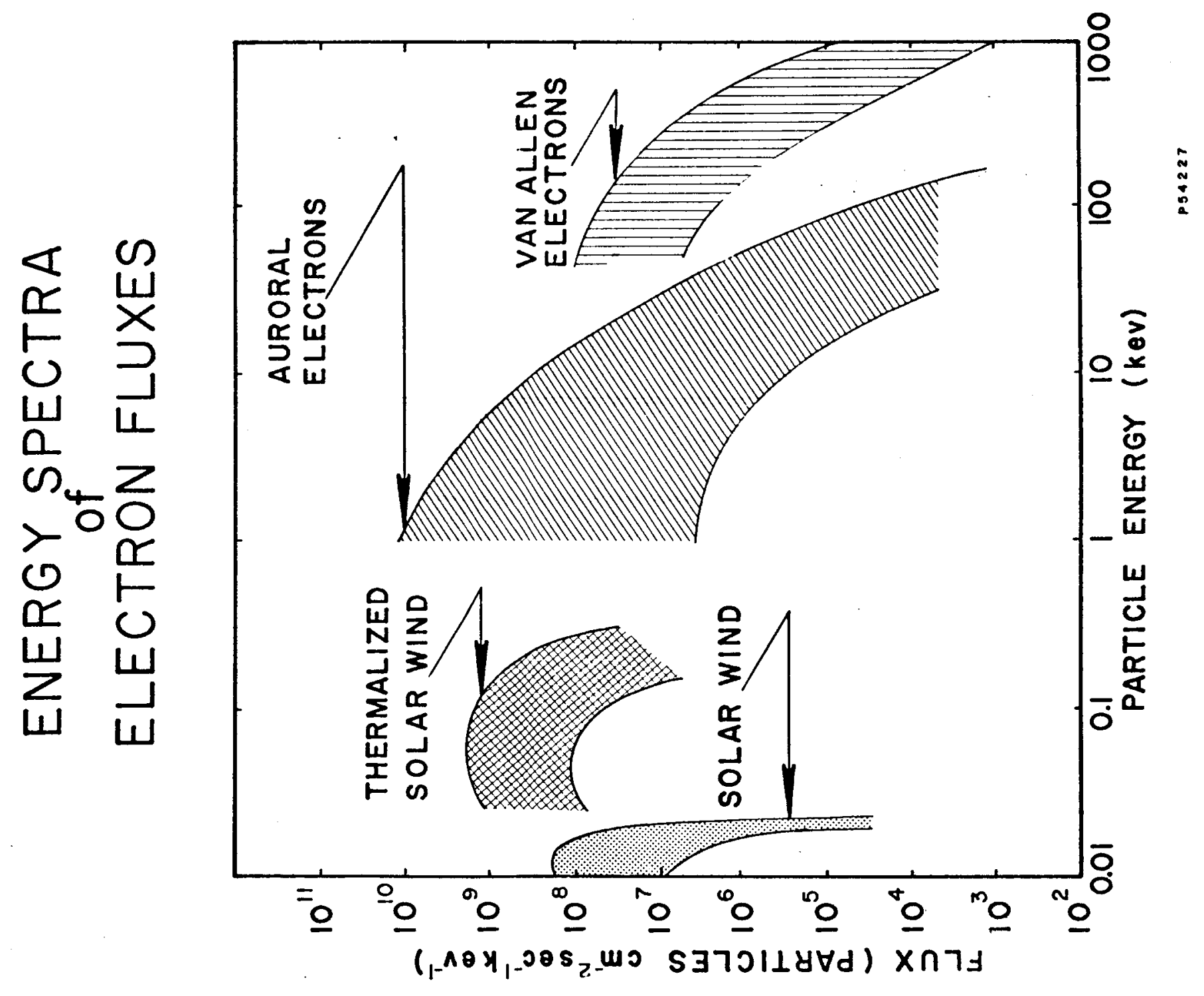




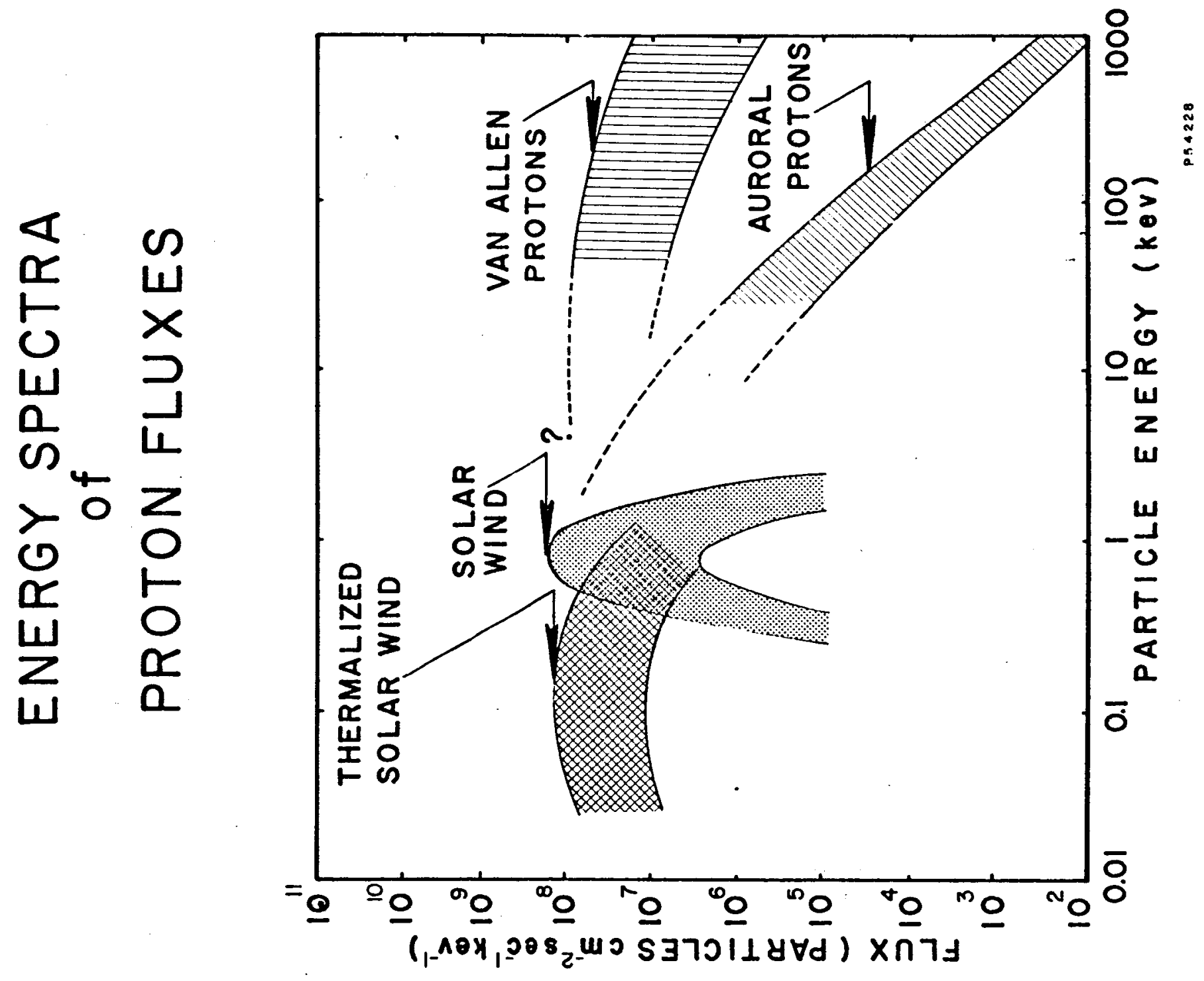

